\title{
Curcumin synergizes with resveratrol to stimulate the MAPK signaling pathway in human articular chondrocytes in vitro
}

\author{
Mehdi Shakibaei $\cdot$ Ali Mobasheri $\cdot$ Constanze Buhrmann
}

Received: 3 February 2010/Accepted: 11 May 2010/Published online: 25 May 2010

(C) Springer-Verlag 2010

\begin{abstract}
The mitogen-activated protein kinase (MAPK) pathway is stimulated in differentiated chondrocytes and is an important signaling cascade for chondrocyte differentiation and survival. Pro-inflammatory cytokines such as interleukin $1 \beta$ (IL-1 $\beta$ ) play important roles in the pathogenesis of osteoarthritis (OA) and rheumatoid arthritis (RA). In this study, we investigated whether curcumin and resveratrol can synergistically inhibit the catabolic effects of IL- $1 \beta$, specifically the inhibition of the MAPK and subsequent apoptosis in human articular chondrocytes. Chondrocytes were either left untreated or treated with $10 \mathrm{ng} / \mathrm{ml} \mathrm{IL-1} \beta$ or $1 \mu \mathrm{M}$ U0126, a specific inhibitor of MAPK pathway alone for the indicated time periods or pretreated with $10 \mu \mathrm{M}$ curcumin, $10 \mu \mathrm{M}$ resveratrol or $10 \mu \mathrm{M}$ resveratrol and $10 \mu \mathrm{M}$ curcumin for $4 \mathrm{~h}$ followed by cotreatment with $10 \mathrm{ng} / \mathrm{ml} \mathrm{IL}-1 \beta$ or $1 \mu \mathrm{M}$ U0126 and $10 \mu \mathrm{M}$ resveratrol, $10 \mu \mathrm{M}$ curcumin or $10 \mu \mathrm{M}$ resveratrol and $10 \mu \mathrm{M}$ curcumin for the indicated time periods. Cultures were evaluated by immunoblotting and transmission electron microscopy. Incubation of chondrocytes with IL- $1 \beta$ resulted in induction of apoptosis, downregulation of $\beta 1$-integrins and the extracellular signal-regulated kinase 1/2 (Erk1/2). Interestingly, U0126 induced apoptosis and
\end{abstract}

M. Shakibaei $(\bowtie) \cdot$ C. Buhrmann

Musculoskeletal Research Group, Institute of Anatomy, Ludwig-Maximilians-University Munich, Pettenkoferstrasse 11, 80336 Munich, Germany

e-mail: mehdi.shakibaei@med.uni-muenchen.de

\section{A. Mobasheri}

Musculoskeletal Research Group, Division of Veterinary

Medicine, School of Veterinary Medicine and Science,

University of Nottingham, Sutton Bonington Campus,

Sutton Bonington LE12 5RD, Leicestershire, UK blocked the above-mentioned proteins in a similar way to IL-1 $\beta$. Furthermore, curcumin and resveratrol inhibited IL-1 $\beta$ - or U0126-induced apoptosis and downregulation of $\beta 1$-integrins and Erk1/2 in human articular chondrocytes. These results suggest that combining these two natural compounds activates MEK/Erk signaling, a pathway that is involved in the maintenance of chondrocyte differentiation and survival.

Keywords Chondrocyte - MAPK pathway - Integrins · OsteoArthritis · IL-1 $\beta$ - Curcumin - Resveratrol

\section{Introduction}

Pro-inflammatory cytokines produced from activated synoviocytes and articular chondrocytes promote degradation of extracellular matrix components [21, 22]. Several lines of evidence suggest that cytokines, such as IL- $1 \beta$ and TNF- $\alpha$, are produced in high quantities in OA and RA in cartilage. Once released, they stimulate the synthesis of more pro-inflammatory cytokines, catabolic factors such as matrix metalloproteinases (MMPs) [22, 39, 40] and mediators of inflammation such as prostaglandins produced by cyclooxygenase-2 (COX-2) [28]. Pro-inflammatory cytokines also induce chondrocyte apoptosis [11], a process that is thought to play a pivotal role in joint diseases (OA and RA) in humans and animals [3, 24, 25]. It has been reported that cytokine-mediated chondrocyte apoptosis can be induced by different signals such as caspase-3, cytochrome-c release, protein kinases and reactive oxygen species (ROS) [38, 50]. Activation of certain caspases such as caspase-3 plays a pivotal role in initiating apoptosis. However, the mechanism of chondrocyte apoptosis in joint diseases has not been fully elucidated. 
Cell-matrix interactions in cartilage are essential for the differentiation and survival of chondrocytes and are mediated by specific receptors e.g. integrins $[18,19,35,45$, 46]. Integrins play a crucial role in early cartilage differentiation, since the presence of anti-integrin antibodies can inhibit differentiation of blastemal cells to chondroblasts [43]. This interaction mediates the activation of the MAPK pathways [33]. Studies from our laboratory have shown that loss of chondrogenic potential is accompanied by reduced expression in key signaling proteins of the mitogen-activated proteins (MAP) kinase (MAPK) pathway and apoptosis [41, 42]. Specific inhibition of the MAPK pathway leads to apoptosis of human chondrocytes in vitro [49]. Furthermore, activation of Sox-9, the chondrogenic transcription factor, seems to be mediated by the MAPK pathway $[16,37]$. It has been shown that cytokines partially reduce Sox-9 protein levels through an NF- $\kappa$ B-dependent pathway in mouse chondrocytes [37, 52] demonstrating how active transcription factors, sharing common co-factors, regulate gene expression. Interestingly, naturally occurring polyphenolic compounds, such as curcumin and resveratrol, are potent agents for modulating inflammation mediating their effects in part by targeting the NF- $\kappa \mathrm{B}$ signaling pathway [10, 44].

Resveratrol (trans-3, 4'-trihydroxystilbene) is a natural phytoalexin that is found in the skin of red grapes, cranberries, peanuts and root extracts of the weed Polygonum cuspidatum [7]. Several lines of evidence indicate that Resveratrol is also capable of immunomodulatory, antioxidative, anti-apoptotic and anti-inflammatory functions [14, 20, 29, 44]. Curcumin (diferuloylmethane), a dietary non-toxic pigment in curry, acts as a potent inhibitor of nuclear transcription factor $\kappa \mathrm{B}$ (NF- $\kappa \mathrm{B})$-activation in several cell types $[10,47,53]$. Curcumin inhibits the constitutive $\mathrm{I} \kappa \mathrm{B} \alpha$ phosphorylation through the inhibition of I $\kappa$ B-kinase (IKK) $[9,10,15,31,47]$. It has been shown that dietary supplements and herbal remedies are capable of suppressing the NF- $\kappa$ B pathway and seem to have positive effects in arthritis therapy; furthermore, it has been reported that curcumin is a potent anti-inflammatory and anticancer compound [2].

Evidence is accumulating to support the idea that signaling pathways malfunction in chondrocytes and synovial cells in aging and well as joint diseases such as OA and RA. Treatment of OA with novel agents that can simultaneously target multiple cellular signaling pathways in chondrocytes will benefit from effectively downregulating inflammation without adverse systemic effects. We and others have shown that phytochemicals such as curcumin and resveratrol target the catabolic pathways mediated by the NF- $\kappa \mathrm{B}$ signal transduction pathway in cartilage and might be used as clinically safe nutritional factors for the treatment of OA [13, 15, 26, 34, 36, 47, 50, 54]. We have also shown that resveratrol and/or curcumin stimulate Sox-9 expression and inhibit the IL- $1 \beta$-induced decreased Sox-9 expression that is necessary for the expression of cartilage matrix genes [15]. Therefore, the aim of the present study was to focus on the MAPK signaling pathway in human chondrocytes and examine the effects of resveratrol and curcumin in combination and in isolation on IL- $1 \beta$-mediated cellular responses.

\section{Materials and methods}

Antibodies

Monoclonal anti- $\beta 1$-integrin, alkaline phosphatase-linked sheep anti-mouse and sheep anti-rabbit secondary antibodies were obtained from Chemicon International (Temecula, CA, USA). Antibody to $\beta$-actin was from Sigma (Munich, Germany). Antibodies to phospho-p42/p44 Erk1/2 and U0126 were purchased from Promega (Mannheim, Germany). Antipan Erk1/2 antibodies were purchased from Transduction Laboratories (Heidelberg, Germany). Polyclonal antibody to active caspase-3 was from R\&D System (Heidelberg, Germany). All antibodies were used at concentrations and dilutions recommended by the manufacturer (dilutions ranged from 1:100 to 1:10,000 for western blot analysis).

\section{Growth medium and chemicals}

Growth medium (Ham's F-12/Dulbecco's modified Eagle's medium (50/50) containing $10 \%$ fetal calf serum (FCS), $25 \mu \mathrm{g} / \mathrm{ml}$ ascorbic acid, $50 \mathrm{IU} / \mathrm{ml}$ streptomycin, $50 \mathrm{IU} / \mathrm{ml}$ penicillin, $2.5 \mu \mathrm{g} / \mathrm{ml}$ amphotericin $\mathrm{B}$, essential amino acids and L-glutamine) was obtained from Seromed (Munich, Germany). Trypsin/EDTA (EC 3.4.21.4) was purchased from Sigma (Munich, Germany). Epon was obtained from Plano (Marburg, Germany). Resveratrol was purchased from Sigma. Curcumin was purchased from Indsaff (Punjab, India). Resveratrol was prepared as a $100 \mathrm{mg} / \mathrm{ml}$ solution in ethanol and then further diluted in cell culture medium. Curcumin was diluted in DMSO as a 5,000 $\mu \mathrm{M}$ concentration and then further diluted in cell culture medium. IL-1 $\beta$ was obtained from Strathman Biotech GmbH (Hannover, Germany).

\section{Chondrocyte isolation and culture}

Cartilage samples from healthy femoral head articular cartilage obtained during joint replacement surgery for femoral neck fractures were used to isolate primary human articular chondrocytes [46]. Cartilage slices were digested primarily with $1 \%$ pronase for $2 \mathrm{~h}$ at $37^{\circ} \mathrm{C}$ and subsequently with $0.2 \%(\mathrm{v} / \mathrm{v})$ collagenase for $4 \mathrm{~h}$ at $37^{\circ} \mathrm{C}$. 
Primary chondrocytes were cultured at a density of 200,000 cells per $60-\mathrm{mm}$ petri dish in monolayer culture for a period of $24 \mathrm{~h}$ at $37^{\circ} \mathrm{C}$ with $5 \% \mathrm{CO}_{2}$. Cartilage samples were derived from human patients with full informed consent and local ethics committee's approval.

\section{Experimental design}

To see the effects of pro-inflammatory cytokines in the absence of any other stimulation caused by serum growth factor, primary human articular chondrocytes were washed three times with serum-starved medium $(0.5 \%$ FCS $)$ and incubated for $1 \mathrm{~h}$ with serum-starved medium. Serumstarved human articular chondrocytes were either left untreated or treated with $10 \mathrm{ng} / \mathrm{ml}$ IL- $1 \beta$, or $1 \mu \mathrm{M}$ U0126 alone for the indicated time periods or pre-treated with $10 \mu \mathrm{M}$ resveratrol, $10 \mu \mathrm{M}$ curcumin or $10 \mu \mathrm{M}$ resveratrol and $10 \mu \mathrm{M}$ curcumin for $4 \mathrm{~h}$ followed by co-treatment with $10 \mathrm{ng} / \mathrm{ml} \mathrm{IL}-1 \beta$ and $10 \mu \mathrm{M}$ resveratrol, $10 \mu \mathrm{M}$ curcumin or $10 \mu \mathrm{M}$ resveratrol and $10 \mu \mathrm{M}$ curcumin for $24 \mathrm{~h}$ or for the indicated time periods.

\section{Transmission electron microscopy}

Chondrocytes were fixed $1 \mathrm{~h}$ with Karnovsky's fixative followed by post-fixation in $1 \% \mathrm{OsO}_{4}$ solution $(0.1 \mathrm{M}$ phosphate buffer). Monolayer cell pellets were rinsed and dehydrated in an ascending alcohol series before being embedded in Epon and cut on a Reichert-Jung Ultracut E (Heidelberg, Germany). Ultrathin sections were contrasted with $2 \%$ uranyl acetate/lead citrate. A transmission electron microscope (TEM 10, Zeiss, Jena, Germany) was used to examine the cultures.

Electron microscopic evaluation of apoptotic cell death

Serum-starved human chondrocytes were exposed to $10 \mathrm{ng} / \mathrm{ml} \mathrm{IL-1} \beta$ or $1 \mu \mathrm{M}$ U0126 alone for $1,12,24$ and $48 \mathrm{~h}$ or pre-stimulated with $10 \mu \mathrm{M}$ resveratrol, $10 \mu \mathrm{M}$ curcumin alone or $10 \mu \mathrm{M}$ resveratrol and $10 \mu \mathrm{M}$ curcumin for $4 \mathrm{~h}$ and then co-treated with IL-1 $\beta$ (10 ng/ml) or U0126 $(1 \mu \mathrm{M})$ for $1,12,24$ and $48 \mathrm{~h}$. Ultra-thin sections of the samples were prepared and evaluated with an electron microscope (TEM 10, Zeiss, Jena, Germany). For statistical analysis, the number of cells with morphological features of apoptotic cell death was determined by scoring 100 cells from 20 different microscopic fields.

\section{Western blot analysis}

To determine the effect of resveratrol/curcumin on IL- $1 \beta$ or U0126-induced apoptosis and inhibition of MAPK signaling pathway, whole cell lysates of chondrocyte monolayers were prepared [44, 46, 49] and equal quantities (500 ng of protein per lane) of total proteins were separated by $\operatorname{SDS}$-PAGE $(5,7.5,12 \%$ gels) under reducing conditions. The separated proteins were transferred onto nitrocellulose membranes. Membranes were pre-incubated in blocking buffer $(5 \%(\mathrm{w} / \mathrm{v})$ skimmed milk powder in PBS/ $0.1 \%$ Tween 20 ) for $1 \mathrm{~h}$ and incubated with primary antibodies against $\beta 1$-integrin, pan-Erk1/2, phospho-Erk1/2, caspase- 3 and $\beta$-actin (overnight, $4^{\circ} \mathrm{C}$ ). Membranes were washed three times with blocking buffer and incubated with alkaline phosphatase-conjugated secondary antibodies for $30 \mathrm{~min}$. They were finally washed three times in $0.1 \mathrm{M}$ Tris pH 9.5 containing $0.05 \mathrm{M} \mathrm{MgCl}_{2}$ and $0.1 \mathrm{M} \mathrm{NaCl}$. Nitro blue tetrazolium and 5-bromo-4-chloro-3-indoylphosphate (p-toluidine salt; Pierce, Rockford, IL, USA) were used as substrates to reveal alkaline phosphatase-conjugated specific antigen-antibody complexes. The density (specific binding) of each band was measured by densitometry using "Quantity One" (Bio-Rad Laboratories Inc. CA, USA).

Statistical analysis

The results are expressed as the means \pm SD of a representative experiment performed in triplicate. The means were compared using student's $t$-test assuming equal variances. $P<0.05$ was considered statistically significant.

\section{Results}

Resveratrol and curcumin suppress IL- $1 \beta$-induced apoptosis in chondrocytes

The effect of curcumin and resveratrol on U0126- or IL- $1 \beta$ induced apoptosis in chondrocytes was examined by transmission electron microscopy. Control monolayer chondrocytes exhibited flattened shapes with small cytoplasmic processes, large and mostly euchromatic nuclei with nucleoli and well-structured cellular organelles (Fig. 1a, A). Serum-starved primary isolated human chondrocytes were treated with $10 \mathrm{ng} / \mathrm{ml} \mathrm{IL-1} \beta$ alone for $1,12,24$ and $48 \mathrm{~h}$ led to degenerative morphological changes (Figs. 1a, B-E), swelling of the rough endoplasmic reticulum, swollen mitochondria (Inset, Fig. 1a, C) and degeneration of other cellular organelles. After longer incubation periods, further cellular degeneration occurred, which included condensation of heterochromatin in cell nuclei and formation of multiple vacuoles. The flattened monolayer chondrocytes became more spherical, lost their microvilli-like processes and underwent apoptosis (Fig. 1a, D-E).

Work from our group has previously demonstrated that U0126 suppresses the MAPK pathway and leads to apoptosis [49]. To determine whether curcumin and resveratrol 


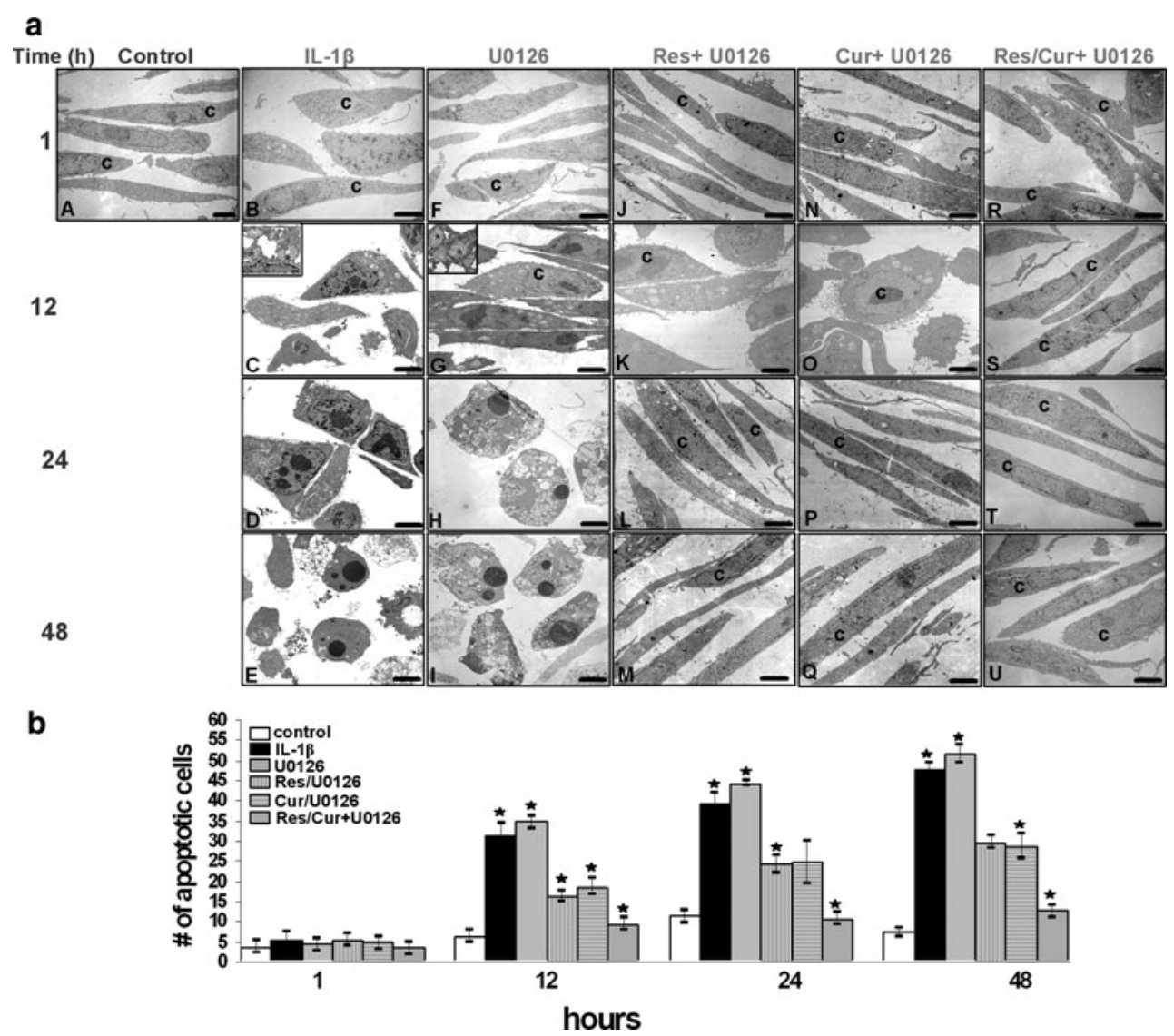

Fig. 1 Effect of resveratrol/curcumin on IL-1 $\beta$ - or U0126-induced degenerative changes and apoptosis in primary chondrocytes. a Electron microscopic demonstration of an untreated chondrocyte $(c)$ containing smooth surface, large nucleus with much loosely packed, functionally active euchromatin and little condensed, functionally inactive heterochromatin, numerous cavities of rough endoplasmic reticulum $(A)$. Treatment with $10 \mathrm{ng} / \mathrm{ml} \mathrm{IL}-1 \beta(B-E)$ or $1 \mu \mathrm{M}$ U0126 $(F-I)$ for $1,12,24,48 \mathrm{~h}$ resulted in nuclear changes with peripheral segregation and aggregation of chromatin into dense areas along the nuclear membrane, swelling and dilatations of cell organelles (mitochondria and endoplasmic reticulum) $(C, G$, Inset $)$ and membrane blebbing. Longer incubations of 24-48 $\mathrm{h}$ led to the formation of apoptotic bodies and cell lysis. However, pre-treatment with $10 \mu \mathrm{M}$

can act synergistically to modulate the cytotoxic effects of U0126 in human chondrocytes, serum-starved chondrocytes were treated with $1 \mu \mathrm{M}$ U0126 alone for $1,12,24$ and $48 \mathrm{~h}$ or were pre-stimulated with $10 \mu \mathrm{M}$ resveratrol, $10 \mu \mathrm{M}$ curcumin or a combination of phytochemicals (each $10 \mu \mathrm{M}$ ) for $4 \mathrm{~h}$ and followed by co-treatment with U0126 $1 \mu \mathrm{M}$ for the indicated time periods. After 12-h treatment with U0126 alone, chondrocytes exhibited morphological features typical of apoptosis, with nuclear changes including chromatin condensation into dense areas along the nuclear membrane and nuclear fragmentation. The cellular membrane was irregular (blebbing), and cytoplasmic vacuoles (dilation of mitochondria and the endoplasmic reticulum) were observed (Figs. 1a, F-I). resveratrol $(J-M)$ or $10 \mu \mathrm{M}$ curcumin $(N-Q)$ alone or with $10 \mu \mathrm{M}$ resveratrol and $10 \mu \mathrm{M}$ curcumin $(R-U)$ followed by co-treatment with U0126 or IL- $1 \beta$ inhibited the adverse effects of IL-1 $\beta$ (data not shown) or U0126 $(J-U)$. After 48-h treatment, chondrocytes demonstrate large, flattened cells with numerous microvilli-like processes, mitochondria and endoplasmic reticulum comparable to control cultures. b To quantify apoptosis in these cultures, 100 cells from 20 microscopic fields were counted. The number of apoptotic cells was highest in cultures stimulated with IL-1 $\beta$ or U0126 alone. However, pre-treatment of the chondrocytes with resveratrol, curcumin or a combination of the two phytochemicals significantly decreased the number of IL-1 $\beta$ - or U0126-induced apoptotic cells compared to each chemical by itself (asterisk)

Interestingly, pre-treatment of the chondrocytes with resveratrol (Figs. 1a, J-M) or curcumin (Figs. 1a, N-Q) alone or in combination (Figs. 1a, R-U) followed by cotreatment with U0126 or IL-1 $\beta$ inhibited the adverse effects of IL-1 $\beta$ (data not shown) or U0126 (Fig. 1a, J-U). After $48 \mathrm{~h}$, chondrocytes demonstrated less cellular degeneration at the ultrastructural level; they regained a flattened shape and exhibited numerous microvilli-like cytoplasmic processes (Figs. 1a, J-U).

Statistical evaluation of apoptotic cell death was carried out by counting the number of apoptotic cells in selected fields from the transmission electron micrographs. This clearly highlighted changes in the number of cells with apoptotic features before and after IL-1 $\beta$ or U0126 
treatment. Pre-treatment with resveratrol and curcumin clearly decreased the number of cells with apoptotic features compared to cells treated with IL- $1 \beta$ or U0126 alone (Fig. 1b). However, pre-treatment of the chondrocytes with a combination of the two phytochemicals decreased the total number of apoptotic cells compared to experiments where each chemical was used in isolation. These results demonstrate that curcumin and resveratrol inhibit the cytotoxic and apoptotic effects induced by IL- $1 \beta$ or U0126 in human chondrocytes.

Resveratrol and curcumin block U0126-induced stimulation of activated caspase- 3 and inhibition of $\beta 1$-integrins in chondrocytes

Previous work from our laboratory has shown that U0126 leads to a marked dose-dependent increase in caspase-3 activation in chondrocytes [49]. To determine whether curcumin and resveratrol can act synergistically to modulate the cytotoxic effects of U0126 in human chondrocytes, serum-starved cells were treated with $1 \mu \mathrm{M}$ U0126 alone for $1,12,24$ and $48 \mathrm{~h}$ or were pre-stimulated with $10 \mu \mathrm{M}$ resveratrol and $10 \mu \mathrm{M}$ curcumin or a combination of phytochemicals (each $10 \mu \mathrm{M}$ ) for $4 \mathrm{~h}$ followed by cotreatment with U0126 $1 \mu \mathrm{M}$ for the indicated time periods. As shown in Fig. 2a, pre-treatment with resveratrol and curcumin significantly downregulated the levels of biologically active caspase-3 in U0126-stimulated cultures compared with primary human chondrocytes stimulated with U0126 alone.
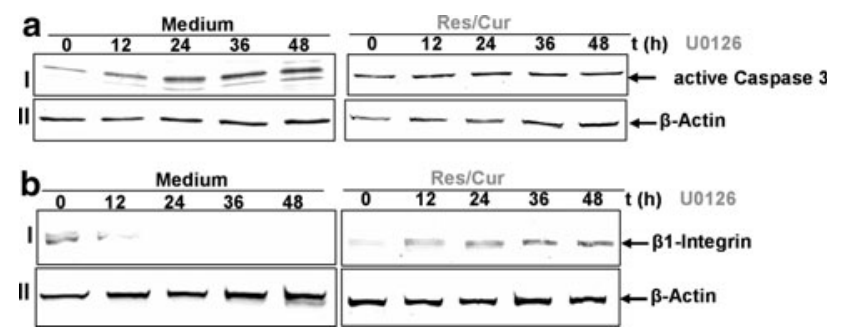

Fig. 2 Resveratrol and curcumin inhibit U0126-induced stimulation of activated caspase-3 and degradation of integrin in primary chondrocytes. Serum-starved human articular chondrocytes were either stimulated for $0,12,24,36,48 \mathrm{~h}$ with $10 \mathrm{ng} / \mathrm{ml} \mathrm{IL-1} \beta, 1 \mu \mathrm{M}$ U0126 or pre-treated with $10 \mu \mathrm{M}$ resveratrol and $10 \mu \mathrm{M}$ curcumin for $4 \mathrm{~h}$ followed by 0-, 12-, 24-, 36-, 48-h stimulation with $10 \mathrm{ng} / \mathrm{ml} \mathrm{IL-}$ $1 \beta$ or $1 \mu \mathrm{M}$ U0126. Equal amounts (500 ng protein per lane) of total proteins were separated by 12 and $7.5 \%$ SDS-PAGE followed by immunoblotting with anti-active caspase- 3 and anti- $\beta 1$-integrin antibodies. A time-dependent downregulation of $\beta 1$-integrin and stimulation of activated caspase- 3 expression was observed. In contrast, pre-treatment with resveratrol and curcumin resulted in a time-dependent increase in $\beta 1$-integrin expression and inhibition of activated caspase-3. Synthesis of the housekeeping protein $\beta$-actin remained unaffected. Data shown are representative of three independent experiments
It is well known that cell-matrix interactions are primarily mediated via multi-functional $\beta 1$-integrins [12]. $\beta 1$-integrins organize cell surface mechanoreceptor complexes and function as signal transduction molecules $[6,35]$ capable of stimulating the MAP kinase pathway [33, 46, 49]. To determine whether curcumin and resveratrol can act synergistically to modulate the inhibitory effects of U0126 on $\beta 1$-integrin expression, serum-starved human chondrocytes were pre-stimulated with a combination of resveratrol and curcumin for $4 \mathrm{~h}$ and co-treated with U0126 for the indicated time periods and then examined by western blotting. As shown in Fig. 2b, U0126 inhibited the expression of $\beta 1$-integrins in a time-dependent manner. In contrast, combination of resveratrol and curcumin stimulated $\beta 1$-integrin expression and inhibited the U0126induced decreased $\beta 1$-integrin expression.

Resveratrol and curcumin prevent U0126-induced inhibition of Erk 1/2 in a dose- and time-dependent manner in chondrocytes

Our group has previously demonstrated that U0126 suppresses the MAPK pathway, which leads to apoptosis [49]. To examine whether resveratrol and curcumin block the U0126-induced inhibition of Erk1/2, serum-starved chondrocytes were probed with anti-pan Erk1/2 antibody and anti-phospho Erk1/2 after pre-treatment with $10 \mu \mathrm{M}$ resveratrol and $10 \mu \mathrm{M}$ curcumin for the indicated times followed by $1 \mu \mathrm{M}$ U0126 stimulation for $30 \mathrm{~min}$ (Fig. 3a). Anti-pan Erk1/2 antibody recognizes both inactive and active forms of Erk1/2 and indicates the expression level of total Erk1/2. Anti-phospho Erk1/2 recognizes only phosphorylated Erk1/2 and indicates the activation of Erk1/2. Furthermore, chondrocytes were pre-incubated with the indicated concentrations of resveratrol and curcumin for $4 \mathrm{~h}$ followed by co-treatment with $1 \mu \mathrm{M}$ U0126 and resveratrol and curcumin for $30 \mathrm{~min}$ (Fig. 3b). As shown in Fig. 3a and b, resveratrol and curcumin stimulated U0126induced Erk1/2 inhibition in a time- (Fig. 3a-I) and dosedependent manner (Fig. 3b-I). In contrast, western blot analysis with a pan-Erk1/2 antibody that recognizes both the phosphorylated and non-phosphorylated forms of Erk1 and 2 confirmed that they did not change in response to U0126 treatment (Fig. 3a-II, b-II).

Resveratrol and curcumin block IL- $1 \beta$ - or U0126induced inhibition of Erk1/2 in chondrocytes

To test whether inhibition of IL-1 $\beta$ - or U0126-induced inhibition of Erk1/2 is blocked by curcumin and resveratrol, serum-starved human articular chondrocytes were prestimulated with $10 \mu \mathrm{M}$ resveratrol and/or $10 \mu \mathrm{M}$ curcumin alone for $12 \mathrm{~h}$. Additionally, serum-starved human 


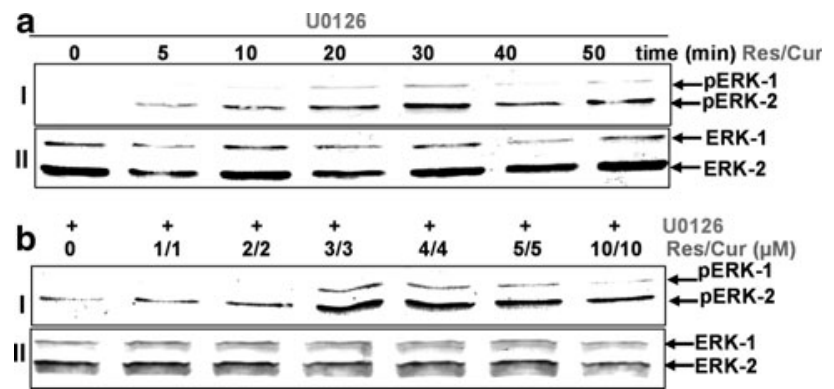

Fig. 3 Resveratrol and curcumin inhibit U0126-induced inhibition of Erk1/2 in primary chondrocytes. a Western blot analysis with U0126treated chondrocytes. Serum-starved chondrocytes were pre-incubated with $10 \mu \mathrm{M}$ resveratrol and $10 \mu \mathrm{M}$ curcumin for $5,10,20,30$, 40 and $50 \mathrm{~min}$, co-treated with $1 \mu \mathrm{M}$ U0126 for $30 \mathrm{~min}$ and then probed (500 ng of protein per lane) for the anti-pan Erk1/2 antibody (control) and anti-phospho Erk1/2. Resveratrol and curcumin pretreatment inhibited U0126-induced Erk1/2 inhibition in a timedependent manner. Synthesis of pan Erk1/2 remained unaffected (a-I, II). b Serum-starved human chondrocytes were pre-incubated with equal concentrations of resveratrol and curcumin $(0,1,2,3,4,5$ and $10 \mu \mathrm{M}$ ) for $4 \mathrm{~h}$ followed by stimulation with $1 \mu \mathrm{M} \mathrm{U} 0126$ for $30 \mathrm{~min}$. The extracts were probed (500 ng of protein per lane) for phospho Erk1/2 by western blot analysis using antibodies to anti-pan Erk1/2 antibody (control) and anti-phospho Erk1/2. A dose-dependent activation of $\mathrm{pErk} 1 / 2$ was observed. The activation of $\mathrm{pErk} 1 / 2$ by resveratrol and curcumin is therefore dose- as well as time-dependent. Synthesis of pan Erk1/2 remained unaffected in nuclear extracts (b-I, II). Data shown are representative of three independent experiments

articular chondrocytes were pre-stimulated with $10 \mu \mathrm{M}$ resveratrol and/or $10 \mu \mathrm{M}$ curcumin alone for $4 \mathrm{~h}$ and then co-treated with IL-1 $\beta$ (10 ng/ml) and/or $1 \mu \mathrm{M} \mathrm{U} 0126$ for $8 \mathrm{~h}$. Some cultures were left untreated and evaluated after $12 \mathrm{~h}$. The activation of pErk $1 / 2$ in the chondrocytes was determined by western blot analysis using anti-pan Erk1/2 antibody and anti-phospho Erk1/2 antibodies. As shown in Fig. 4I, IL-1 $\beta$ - or U0126-induced inhibition of pErk1/2 was blocked by curcumin and/or resveratrol. In contrast, western blot analysis with a pan-Erk antibody that recognizes both the phosphorylated and non-phosphorylated forms of Erk1 and 2 did not change in response to IL- $1 \beta$ or U0126 treatment (Fig. 4II).

Taken together, these results suggest that resveratrol and curcumin inhibit the cytotoxic and apoptotic effects induced by IL- $1 \beta$ or U0126, at least in part by activation of MAPK pathway in human chondrocytes.

\section{Discussion}

In this paper, we investigated the synergistic effects of resveratrol and curcumin on apoptotic signaling in IL- $1 \beta$ stimulated human chondrocytes. The present study leads to the following findings: (1) treatment of chondrocytes with $10 \mathrm{ng} / \mathrm{ml} \mathrm{IL}-1 \beta$ or $1 \mu \mathrm{M}$ U0126, a specific inhibitor of the

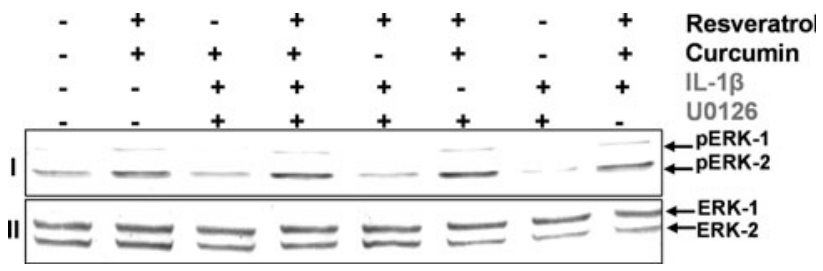

Fig. 4 Effects of Resveratrol/Curcumin on IL-1 $\beta$-induced inhibition of MAPK pathway in human articular chondrocytes in vitro. Serumstarved human chondrocytes were pre-treated with $10 \mu \mathrm{M}$ resveratrol and/or $10 \mu \mathrm{M}$ curcumin alone for $12 \mathrm{~h}$. Other serum-starved chondrocytes were pre-stimulated with $10 \mu \mathrm{M}$ resveratrol and/or $10 \mu \mathrm{M}$ curcumin alone for $4 \mathrm{~h}$ and then co-treated with IL-1 $\beta(10 \mathrm{ng} / \mathrm{ml})$ or/ and $1 \mu \mathrm{M}$ U0126 for $8 \mathrm{~h}$. Some cultures were left untreated and evaluated after $12 \mathrm{~h}$. Equal amounts (500 ng protein per lane) of total proteins were separated by $12 \%$ SDS-PAGE and analyzed by immunoblotting with anti-pan-ERK and anti-pErk1/2 antibodies. IL-1 $\beta$ - or U0126-induced inhibition of pErk1/2 was blocked by curcumin and/or resveratrol (I). In contrast, western blot analysis with a pan-ERK antibody, which recognizes both the phosphorylated and non-phosphorylated forms of Erk1 and 2, confirms that these did not change in response to U0126 treatment (II). Data shown are representative of three independent experiments

MAPK signaling pathway, which can inhibit the phosphorylation and activation of Erk1/Erk2, results in morphological alterations and apoptosis, which were abolished through pre-treatment with resveratrol and curcumin. (2) U0126 induced an increase in activated caspase- 3 and decrease in $\beta 1$-integrin signal receptor synthesis, and these were relieved by resveratrol and curcumin in combination in a time-dependent manner. (3) Resveratrol potentiates the anti-apoptotic effects of curcumin on IL-1 $\beta$ or U0126stimulated chondrocytes, and this correlates with up-regulation of the MAPK pathway. (4) Additionally, both resveratrol and/or curcumin suppressed U0126-induced downregulation of the p-Erk $1 / 2$ in a dose- and timedependent manner. (5) Finally, IL-1 $\beta$ or U0126 inhibition of activation and phosphorylation of Erk1/2 could be clearly blocked by resveratrol and/or curcumin.

In the present study, we demonstrated that IL- $1 \beta$ and the specific inhibition of the MAPK pathway led to apoptosis and downregulation of $\beta 1$-integrin receptor and Erk1/2 expression in chondrocytes. Our data show for the first time that this process is inhibited when chondrocytes are treated with the natural phytochemicals resveratrol and curcumin.

Chondrocytes synthesize a cartilage-specific pericellular matrix, which consists primarily of type-II collagen and cartilage-specific proteoglycans [43]. This specific extracellular matrix and several additional substances are required for chondrocyte differentiation and survival [17]. Interaction of chondrocytes and their surrounding matrix plays an essential role in maintaining their differentiated phenotype. This is partly mediated by $\beta 1$-integrins, a specific surface receptor and transmembrane signal transduction molecule [18, 32, 43, 46, 48, 49, 51]. Furthermore, 
several lines of evidence suggest that $\beta 1$-integrins stimulate different signal transduction pathways, such as the mitogen-activated protein (MAP) kinase pathway, and thus control proliferation, differentiation and survival of cells $[23,33,55]$.

Erk1/2 regulates the activities of several nuclear transcription factors [27], such as cartilage-specific transcription factor Sox-9, which is an important transcription factor for the expression of chondrocyte-specific marker genes, including collagen types II and XI and cartilage-specific proteoglycans $[4,5]$. Indeed, a specific inhibitor (U0126) of the MAP kinase signaling pathway, which can block the activation of Erk1/2, suppresses the FGF-2-stimulated increase in Sox-9 levels and hence also the transcription of cartilage-specific extracellular matrix genes [37]. Moreover, we have previously shown that the U0126-induced inhibition of the MAP kinase signaling pathway suppresses the activation of Erk1/2 and promotes apoptosis in cultured human chondrocytes, as revealed by the activation of caspase-3 and the cleavage of poly(ADP-ribose)polymerase (PARP) [49].

Pro-inflammatory cytokines such as IL-1 $\beta$ have been shown to mediate cartilage degradation and apoptosis in chondrocytes in degenerative joint diseases such as RA and

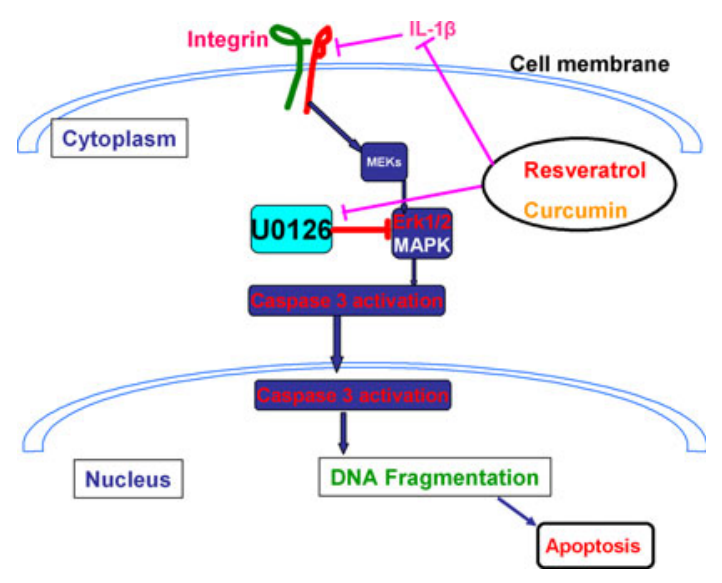

Fig. 5 Molecular model outlining the proposed mechanism for the stimulatory effects of resveratrol and curcumin on IL-1 $\beta$ - or U0126induced MAPK pathway inhibition and apoptosis in primary chondrocytes in vitro. Signal transduction through $\beta 1$-integrins leads to the activation of the Ras-MAP Kinase signaling pathway. Activation of the MAPK pathway may be one of the mechanisms by which $\beta 1$ integrins regulate chondrocyte gene expression. Previous studies have shown that activation of the MAPK pathway regulates the activity of a number of intracellular signaling proteins through phosphorylation. Erk1/Erk2, a downstream kinase of the MAPK pathway, regulates the expression and activity of various transcription factors. Specific inhibition of Erk1/Erk2 by U0126 or IL- $1 \beta$ results in cleavage of caspase-3 in human chondrocytes in vitro. Since activation of caspase-3 and DNA fragmentation are common features of apoptosis, the specific inhibition of the Ras-mitogen-activated kinase leads to chondrocyte apoptosis. Resveratrol and curcumin both block the inhibition effect of U0126 or IL-1 $\beta$ on the MAPK pathway in chondrocytes
OA in humans as well as in animals $[3,21]$. Resveratrol and curcumin are anti-inflammatory phytochemicals that have previously been shown to block some of the catabolic effects of TNF- $\alpha$ and IL- $1 \beta$ via inhibition of NF-kB in different cell types $[1,8,14,30]$. Our previous work on chondrocytes has demonstrated that resveratrol and curcumin specifically inhibit NF-kB through inhibition of the proteasome activity and/or inhibition of IKK activation. This inhibition stimulated anti-apoptotic gene products (Bcl-2, Bcl-xL and TRAF1), suppressed the pro-apoptosis proteins (caspase-3, PARP) and matrix-degrading gene products (MMP-3 and MMP -9) and angiogenesis and inflammation gene products (VEGF and COX-2) $[14,15$, $44,47]$. Apoptosis plays a crucial role in inflammatory processes such as RA and OA, leading to cartilage degradation and joint disease. This study confirms that the naturally occurring polyphenolic compounds resveratrol and curcumin exert synergistic effects on the MAPK signaling pathway. This suggests that the anti-inflammatory and anti-apoptotic effects of resveratrol and/or curcumin are mediated, at least in part, through the MEK/Erk signal transduction pathway (Fig. 5).

Acknowledgments Ms. Christina Pfaff, Ms. Stefanie Schreiber and Ms. Ursula Schwikowski are gratefully acknowledged for their excellent technical assistance.

\section{References}

1. Aggarwal BB (2004) Nuclear factor-kappaB: the enemy within. Cancer Cell 6:203-208

2. Aggarwal BB, Kumar A, Bharti AC (2003) Anticancer potential of curcumin: preclinical and clinical studies. Anticancer Res 23:363-398

3. Aigner T, Kim HA (2002) Apoptosis and cellular vitality: issues in osteoarthritic cartilage degeneration. Arthritis Rheum 46:1986-1996

4. Akiyama H, Chaboissier MC, Martin JF, Schedl A, de Crombrugghe B (2002) The transcription factor Sox 9 has essential roles in successive steps of the chondrocyte differentiation pathway and is required for expression of Sox 5 and Sox6. Genes Dev $16: 2813-2828$

5. Akiyama H, Lyons JP, Mori-Akiyama Y, Yang X, Zhang R, Zhang Z, Deng JM, Taketo MM, Nakamura T, Behringer RR, McCrea PD, de Crombrugghe B (2004) Interactions between Sox 9 and beta-catenin control chondrocyte differentiation. Genes Dev 18:1072-1087

6. Albelda SM, Buck CA (1990) Integrins and other cell adhesion molecules. Faseb J 4:2868-2880

7. Baolin L, Inami Y, Tanaka H, Inagaki N, Iinuma M, Nagai $H$ (2004) Resveratrol inhibits the release of mediators from bone marrow-derived mouse mast cells in vitro. Planta Med 70:305-309

8. Bhardwaj A, Sethi G, Vadhan-Raj S, Bueso-Ramos C, Takada Y, Gaur U, Nair AS, Shishodia S, Aggarwal BB (2007) Resveratrol inhibits proliferation, induces apoptosis, and overcomes chemoresistance through down-regulation of STAT3 and nuclear factorkappaB-regulated antiapoptotic and cell survival gene products in human multiple myeloma cells. Blood 109:2293-2302 
9. Bharti AC, Aggarwal BB (2002) Nuclear factor-kappa B and cancer: its role in prevention and therapy. Biochem Pharmacol 64:883-888

10. Bharti AC, Donato N, Singh S, Aggarwal BB (2003) Curcumin (diferuloylmethane) down-regulates the constitutive activation of nuclear factor-kappa B and Ikappa Balpha kinase in human multiple myeloma cells, leading to suppression of proliferation and induction of apoptosis. Blood 101:1053-1062

11. Blanco FJ, Guitian R, Moreno J, de Toro FJ, Galdo F (1999) Effect of antiinflammatory drugs on COX-1 and COX-2 activity in human articular chondrocytes. J Rheumatol 26:1366-1373

12. Cao L, Lee V, Adams ME, Kiani C, Zhang Y, Hu W, Yang BB (1999) Beta-integrin-collagen interaction reduces chondrocyte apoptosis. Matrix Biol 18:343-355

13. Clutterbuck AL, Mobasheri A, Shakibaei M, Allaway D, Harris $P$ (2009) Interleukin-1beta-induced extracellular matrix degradation and glycosaminoglycan release is inhibited by curcumin in an explant model of cartilage inflammation. Ann N Y Acad Sci 1171:428-435

14. Csaki C, Keshishzadeh N, Fischer K, Shakibaei M (2008) Regulation of inflammation signalling by resveratrol in human chondrocytes in vitro. Biochem Pharmacol 75:677-687

15. Csaki C, Mobasheri A, Shakibaei M (2009) Synergistic chondroprotective effects of curcumin and resveratrol in human articular chondrocytes: inhibition of IL-1beta-induced NF-kappaB-mediated inflammation and apoptosis. Arthritis Res Ther 11:R165

16. de Crombrugghe B, Lefebvre V, Behringer RR, Bi W, Murakami S, Huang W (2000) Transcriptional mechanisms of chondrocyte differentiation. Matrix Biol 19:389-394

17. Dessau W, von der Mark H, von der Mark K, Fischer S (1980) Changes in the patterns of collagens and fibronectin during limbbud chondrogenesis. J Embryol Exp Morphol 57:51-60

18. Durr J, Goodman S, Potocnik A, von der Mark H, von der Mark K (1993) Localization of beta 1-integrins in human cartilage and their role in chondrocyte adhesion to collagen and fibronectin. Exp Cell Res 207:235-244

19. Enomoto M, Leboy PS, Menko AS, Boettiger D (1993) Beta 1 integrins mediate chondrocyte interaction with type I collagen, type II collagen, and fibronectin. Exp Cell Res 205:276-285

20. Estrov Z, Shishodia S, Faderl S, Harris D, Van Q, Kantarjian HM, Talpaz M, Aggarwal BB (2003) Resveratrol blocks interleukin-1beta-induced activation of the nuclear transcription factor NF-kappaB, inhibits proliferation, causes S-phase arrest, and induces apoptosis of acute myeloid leukemia cells. Blood 102:987-995

21. Feldmann M, Brennan FM, Maini RN (1996) Role of cytokines in rheumatoid arthritis. Annu Rev Immunol 14:397-440

22. Fernandes JC, Martel-Pelletier J, Pelletier JP (2002) The role of cytokines in osteoarthritis pathophysiology. Biorheology 39:237246

23. Giancotti FG, Ruoslahti E (1999) Integrin signaling. Science 285:1028-1032

24. Goldring MB (2000) Osteoarthritis and cartilage: the role of cytokines. Curr Rheumatol Rep 2:459-465

25. Hashimoto S, Ochs RL, Komiya S, Lotz M (1998) Linkage of chondrocyte apoptosis and cartilage degradation in human osteoarthritis. Arthritis Rheum 41:1632-1638

26. Jackson JK, Higo T, Hunter WL, Burt HM (2006) The antioxidants curcumin and quercetin inhibit inflammatory processes associated with arthritis. Inflamm Res 55:168-175

27. Kortenjann M, Shaw PE (1995) Raf-1 kinase and ERK2 uncoupled from mitogenic signals in rat fibroblasts. Oncogene 11:2105-2112

28. Largo R, Alvarez-Soria MA, Diez-Ortego I, Calvo E, SanchezPernaute O, Egido J, Herrero-Beaumont G (2003) Glucosamine inhibits IL-1beta-induced NFkappaB activation in human osteoarthritic chondrocytes. Osteoarthritis Cartilage 11:290-298

29. Leiro J, Arranz JA, Parama A, Alvarez MF, Sanmartin ML (2004) In vitro effects of the polyphenols resveratrol, mangiferin and (-)-epigallocatechin-3-gallate on the scuticociliate fish pathogen Philasterides dicentrarchi. Dis Aquat Organ 59:171-174

30. Liacini A, Sylvester J, Li WQ, Huang W, Dehnade F, Ahmad M, Zafarullah M (2003) Induction of matrix metalloproteinase-13 gene expression by TNF-alpha is mediated by MAP kinases, AP1, and NF-kappaB transcription factors in articular chondrocytes. Exp Cell Res 288:208-217

31. Lin JK, Pan MH, Lin-Shiau SY (2000) Recent studies on the biofunctions and biotransformations of curcumin. BioFactors 13:153-158

32. Loeser RF (1993) Integrin-mediated attachment of articular chondrocytes to extracellular matrix proteins. Arthritis Rheum 36:1103-1110

33. Marshall CJ (1994) MAP kinase kinase kinase, MAP kinase kinase and MAP kinase. Curr Opin Genet Dev 4:82-89

34. Mathy-Hartert M, Jacquemond-Collet I, Priem F, Sanchez C, Lambert C, Henrotin Y (2009) Curcumin inhibits pro-inflammatory mediators and metalloproteinase- 3 production by chondrocytes. Inflamm Res 58:899-908

35. Mobasheri A, Carter SD, Martin-Vasallo P, Shakibaei M (2002) Integrins and stretch activated ion channels; putative components of functional cell surface mechanoreceptors in articular chondrocytes. Cell Biol Int 26:1-18

36. Mun SH, Kim HS, Kim JW, Ko NY, Kim DK, Lee BY, Kim B, Won HS, Shin HS, Han JW, Lee HY, Kim YM, Choi WS (2009) Oral administration of curcumin suppresses production of matrix metalloproteinase (MMP)-1 and MMP-3 to ameliorate collageninduced arthritis: inhibition of the PKCdelta/JNK/c-Jun pathway. Journal of pharmacological sciences 111:13-21

37. Murakami S, Lefebvre V, de Crombrugghe B (2000) Potent inhibition of the master chondrogenic factor Sox 9 gene by interleukin-1 and tumor necrosis factor-alpha. J Biol Chem 275:3687-3692

38. Rath PC, Aggarwal BB (1999) TNF-induced signaling in apoptosis. J Clin Immunol 19:350-364

39. Robbins JR, Thomas B, Tan L, Choy B, Arbiser JL, Berenbaum F, Goldring MB (2000) Immortalized human adult articular chondrocytes maintain cartilage-specific phenotype and responses to interleukin-1beta. Arthritis Rheum 43:2189-2201

40. Ryu J, Treadwell BV, Mankin HJ (1984) Biochemical and metabolic abnormalities in normal and osteoarthritic human articular cartilage. Arthritis Rheum 27:49-57

41. Schulze-Tanzil G, de Souza P, Villegas Castrejon H, John T, Merker HJ, Scheid A, Shakibaei M (2002) Redifferentiation of dedifferentiated human chondrocytes in high-density cultures. Cell Tissue Res 308:371-379

42. Schulze-Tanzil G, Mobasheri A, de Souza P, John T, Shakibaei M (2004) Loss of chondrogenic potential in dedifferentiated chondrocytes correlates with deficient Shc-Erk interaction and apoptosis. Osteoarthritis and cartilage/OARS, Osteoarthritis Research Society 12:448-458

43. Shakibaei M (1998) Inhibition of chondrogenesis by integrin antibody in vitro. Exp Cell Res 240:95-106

44. Shakibaei M, Csaki C, Nebrich S, Mobasheri A (2008) Resveratrol suppresses interleukin-1beta-induced inflammatory signaling and apoptosis in human articular chondrocytes: potential for use as a novel nutraceutical for the treatment of osteoarthritis. Biochem Pharmacol 76:1426-1439

45. Shakibaei M, De Souza P, Merker HJ (1997) Integrin expression and collagen type II implicated in maintenance of chondrocyte shape in monolayer culture: an immunomorphological study. Cell Biol Int 21:115-125 
46. Shakibaei M, John T, De Souza P, Rahmanzadeh R, Merker HJ (1999) Signal transduction by beta1 integrin receptors in human chondrocytes in vitro: collaboration with the insulin-like growth factor-I receptor. Biochem J 342 Pt 3:615-623

47. Shakibaei M, John T, Schulze-Tanzil G, Lehmann I, Mobasheri A (2007) Suppression of NF-kappaB activation by curcumin leads to inhibition of expression of cyclo-oxygenase-2 and matrix metalloproteinase-9 in human articular chondrocytes: implications for the treatment of osteoarthritis. Biochem Pharmacol 73:1434-1445

48. Shakibaei M, Mobasheri A (2003) Beta1-integrins co-localize with $\mathrm{Na}, \mathrm{K}$-ATPase, epithelial sodium channels $(\mathrm{ENaC})$ and voltage activated calcium channels (VACC) in mechanoreceptor complexes of mouse limb-bud chondrocytes. Histol Histopathol 18:343-351

49. Shakibaei M, Schulze-Tanzil G, de Souza P, John T, Rahmanzadeh M, Rahmanzadeh R, Merker HJ (2001) Inhibition of mitogen-activated protein kinase kinase induces apoptosis of human chondrocytes. J Biol Chem 276:13289-13294
50. Shakibaei M, Schulze-Tanzil G, Takada Y, Aggarwal BB (2005) Redox regulation of apoptosis by members of the TNF superfamily. Antioxid Redox Signal 7:482-496

51. Shakibaei M, Zimmermann B, Merker HJ (1995) Changes in integrin expression during chondrogenesis in vitro: an immunomorphological study. J Histochem Cytochem 43:1061-1069

52. Sitcheran R, Cogswell PC, Baldwin AS Jr (2003) NF-kappaB mediates inhibition of mesenchymal cell differentiation through a posttranscriptional gene silencing mechanism. Genes Dev $17: 2368-2373$

53. Surh YJ (2003) Cancer chemoprevention with dietary phytochemicals. Nat rev 3:768-780

54. Toegel S, Wu SQ, Piana C, Unger FM, Wirth M, Goldring MB, Gabor F, Viernstein H (2008) Comparison between chondroprotective effects of glucosamine, curcumin, and diacerein in IL-1beta-stimulated C-28/I2 chondrocytes. Osteoarthritis Cartilage 16:1205-1212

55. van der Flier A, Sonnenberg A (2001) Function and interactions of integrins. Cell Tissue Res 305:285-298 